\title{
Implant therapy post sinus lifting in the edentulous maxilla: evidence - based guidelines.
}

\author{
Chalazoniti A., Papavasileiou D., Vlachaki A., Christopoulos P., Kamposiora P. \\ Department of Prosthodontics, Department of Oral \& Maxillofacial Surgery \\ National and Kapodistrian University of Athens, Greece
}

\section{Introduction:}

Treating an edentulous posterior maxilla with implant-supported prostheses can prove to be challenging. In severely atrophic maxillae, alveolar ridge resorption combined with sinus pneumatization leads to inadequate bone volume for standard-length implants, calling for bone augmentation procedures such as sinus floor lifting, when short implants are contraindicated. To ensure correct treatment planning, implant and implant-supported prostheses' success rate should be assessed.

\section{Aim:}

To evaluate the factors affecting the success and survival rate of dental implants placed in edentulous atrophic maxillae that necessitated sinus lifting procedures, and to propose evidence-based guidelines concerning the optimal treatment of such cases.

\section{Materials and Methods:}

An online search of systematic reviews published within the last ten years was conducted using the PubMed database. The titles and abstracts identified were screened using the following inclusion criteria: articles on implant treatment of augmented sinuses in atrophic maxillae with remaining bone height of less than 4-6mm, examining the effect of the surgical technique, the type of the grafting material, implant surface type, implant placement time on the success and survival rate of the restorations.

\section{Results:}

The search yielded 6 systematic reviews published that fulfilled the inclusion criteria and were relevant to the study. The selected reviews are presented in the following table.

\begin{tabular}{|l|l|l|l|l|l|}
\hline Review & Included Papers & RCTs & Non-RCTs & No of patients & No of implants \\
\hline Chao et al, 2010 & 21 & 0 & 9 CTs, 4 Pros, 3 Retro, 5 Other & 789 & 2262 \\
\hline Corbella et al, 2013 & 41 & 1 & 12 Pros, 23 Retro, 5 Other & 4166 & 10498 \\
\hline $\begin{array}{l}\text { Del Fabbro et al, 2013 } \\
\text { Esposito et al, 2014 }\end{array}$ & 24 & 1 & 15 Retro, 5 CSs, 3 CTs & 20853 & 7757 \\
\hline $\begin{array}{l}\text { Pjetursson et al, 2008 } \\
\text { Rios et al, 2009 }\end{array}$ & 14 & 14 & 0 & 548 & NS \\
\hline
\end{tabular}

RCTs: Randomized Controlled Trials, CTs: Clinical Trials, Pros: Prospective Studies, Retro: Retrospective Studies, CSs: Clinical Series, NS: Not Specified

The factors presenting a significant effect on implant prognosis included:

\section{Surgical technique:}

Lateral approach sinus floor elevation (LASFE): The procedure is generally chosen in atrophic maxillae, with an initial alveolar bone height of $\leq 4 \mathrm{~mm}$. Implant survival rate (SR) is allegedly affected by the residual bone height, ranging from $86 \%-96 \%$ at $\leq 4 \mathrm{~mm}$, and $96 \%$ $100 \%$ at $>4 \mathrm{~mm}$.

Osteotome sinus floor elevation (OSFE): The procedure was originally recommended for maxillae with an initial alveolar bone height of $>5 \mathrm{~mm}$. Considered less invasive than LASFE, in $>4 \mathrm{~mm}$ of residual bone height OSFE yields similarly high implant SRs, from 93\% $100 \%$. However, there are insufficient data concerning implant SR in severely atrophic maxillae.

When the residual bone height is $\leq 4 \mathrm{~mm}$, for a more controlled and predictable outcome, LASFE should be preferred over OSFE.

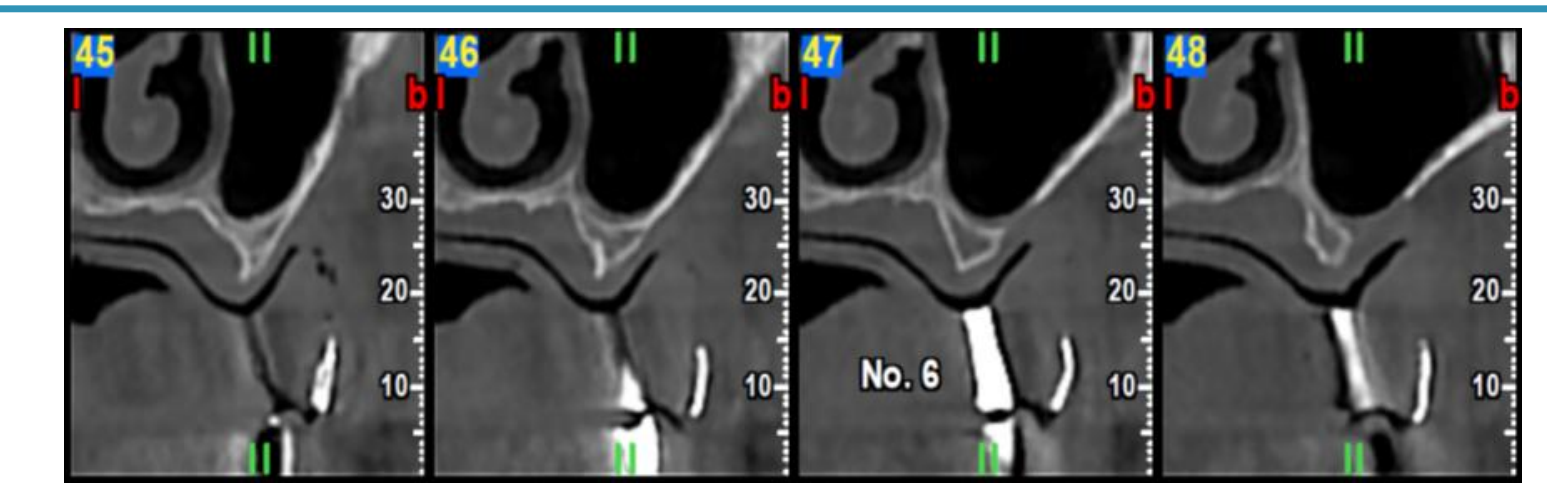

Picture 1: CBCT of a left maxillary sinus with $<4 \mathrm{~mm}$ of initial alveolar bone height. Lack of buccal bone is visible in cuts 47,48 . Treatment of choice is LASFE.

\section{Graft material:}

Grafting materials used for sinus lifting procedures include autogenous bone $(\mathrm{AB})$, bone substitute materials (BSM), or a BSM mixed with $\mathrm{AB}$ at a $20 \%-70 \%$ ratio. No significant differences in implant SR were observed between the three groups (98\% AB, 97\% BSM, 100\% BSM mixed with $\mathrm{AB}$ ).

Implant survival seems to be independent of the grafting biomaterial used.

\section{Membrane coverage:}

The use of a resorbable membrane to cover the grafting material appears to improve implant SR, with the majority of studies showing a statistically significant difference.

Post-operative membrane coverage of the surgical window should be preferred.

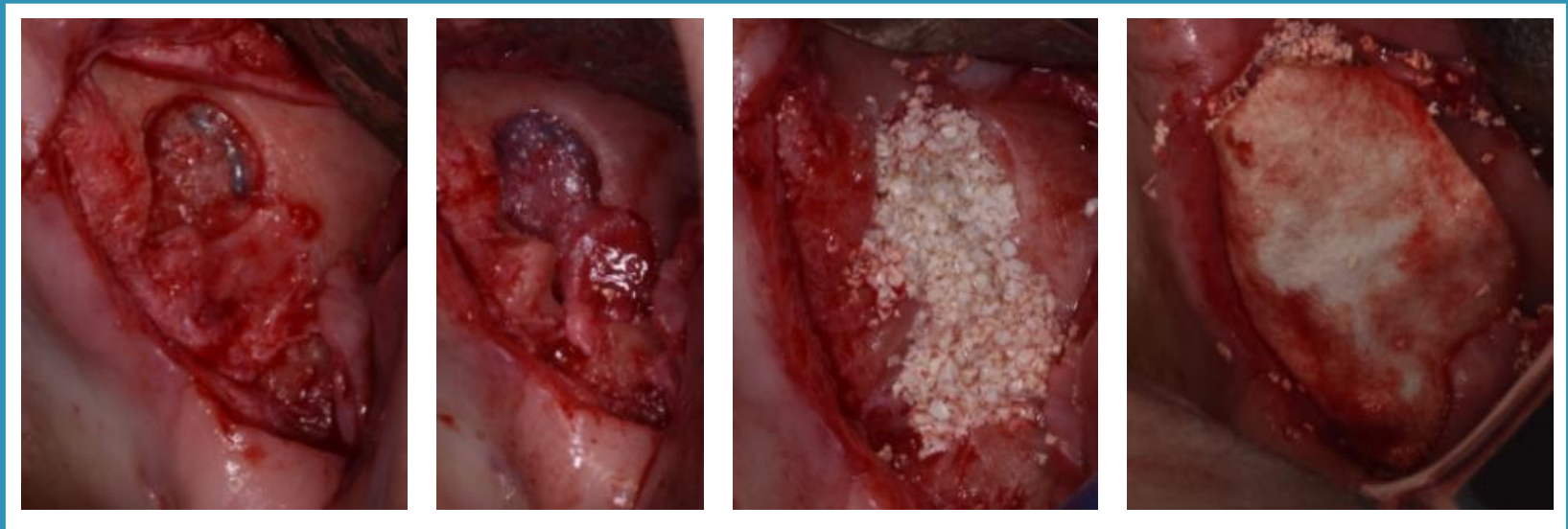

Picture 2: Preparation of the sinus membrane, use of BSM, coverage with resorbable collagen membrane.

\section{Implant placement and surface:}

Both simultaneous and delayed implant placement end in comparable results, with a slightly improved implant SR when a one-stage approach was followed. The use of textured implants led to significantly improved implant SR compared to machined implants.

\section{Rough surface implants should be preferred. \\ A two-stage technique is preferable whenever primary implant stability cannot be achieved.}

\section{Conclusion and Clinical Implications:}

Due to the low number of RCTs included, the level of evidence of the included reviews is not very high. Therefore, more high evidencebased studies are needed to ensure more reliable guidelines, and a predictable outcome.

\section{References:}

- Chao et al, 2010, doi: 10.1111/j.1600-051X.2010.01555.x

- Corbella et al, 2013, doi: 10.1111/cid.12077

- Del Fabbro et al, 2013, doi: 10.11607/prd.1288

- Esposito et al, 2014, doi: 10.1002/14651858.CD008397.pub2.

- Pjetursson et al, 2008, doi: 10.1111/j.1600-051X.2008.01272.x

- Rios et al, 2009, doi: 10.1097/ID.0b013e3181b4af93. 